\title{
Volumetric tools for machining planning
}

\author{
G. Renner and I. Stroud* \\ Computer and Automation Researchc Institute, Hungary \\ renner@sztaki.hu
}

*EPFL, DGM-ICAP-LICP, CH-1015 Lausanne, Switzerland

\begin{abstract}
The paper presents briefly, as an introduction, the MAT calculation method by subdivision, the so-called "divide-and-conquer» algorithm during which the Delaunay nodes are calculated. The paper goes on to describe how this method can be applied to calculating the MAT of negative objects. The negative MAT provides important information about the exterior of the object for material removal and maximal radii for access. The process of calculating the negative MAT includes the determination of infinite Delaunay nodes as well. The paper presents methods for recombining the Delaunay nodes according to certain rules for object reconstruction, offsetting and object subdivision. Finally the paper describes how these tools can be used for machining and machining planning.
\end{abstract}

Key words: MAT, offsetting, volumetric decomposition

\section{Introduction}

Currently the Boundary Representation (B-rep) technique is widely used as a solid model representation technique in $\mathrm{CAD}$ and $\mathrm{CAM}$ systems. However, one of the drawbacks of this technique is that it is essentially local in nature and there is no implicit notion of volumetric characteristics. For many applications, however, it is necessary to have this information. For example, in order to manufacture an object it would inevitably be necessary to have information on volumetric properties such as thickness, clearances etc. It is possible to test selected points to find material thickness, or other characteristics, but these points have to be selected using some, possibly, arbitrary rules and their computation is complicated and time

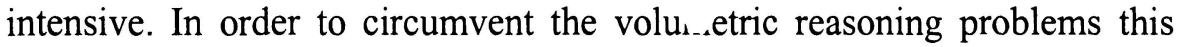

The original version of this chapter was revised: The copyright line was incorrect. This has been corrected. The Erratum to this chapter is available at DOI: 10.1007/978-0-387-35492-7_50 
paper proposes the use of techniques based on the Medial Axis Transform surface (MAT). Techniques for calculation of the MAT have been developed over a period of several years now. A recent development is the use of general topological Delaunay nodes for object reconstruction, offsetting and subdivision. This is a development of the multiple start point algorithm described by Renner and Stroud [ReSt96]. Most algorithms mention only Delaunay triangles (in 2D) and tetrahedra (in 3D) but these are only the minimum structures. These may suffice to delimit a MAT vertex, but do not reflect the structure of the object. The generalised node structure is usually more complex.

The paper presents briefly, as an introduction, the MAT calculation method by subdivision, the so-called «divide-and-conquer» algorithm during which the Delaunay nodes are calculated. The paper goes on to describe how this method can be applied to calculating the MAT of negative objects. The negative MAT provides important information about the exterior of the object for material removal and maximal radii for access. The process of calculating the negative MAT includes the determination of infinite Delaunay nodes. The paper presents methods for recombining the Delaunay nodes according to certain rules for object reconstruction, offsetting and object subdivision. Finally the paper describes how these tools can be used for machining and machining planning. The object decomposition method uses rules to recombine the Delaunay nodes into groups corresponding to volumetric elements.

Offsetting technique are used for the computation of tool paths. In contrast with the usual offsetting process, the MAT-based offsetting is much quicker, because the proximity information is pre-calculated, only the recalculation is needed. This makes it possible for a user to use interactive trial-and-error methods to determine the cutter centre surface. The MAT is easy to use for constant value offsetting. This just uses a simple recombination technique to determine the topology of the offset object(s) and then recomputes the geometry. This is analogous to the $2 \mathrm{~d}$ pocket machining case, where the $2 \mathrm{D}$ Voronoi diagram can be used for fast calculation of toolpaths.

\section{Divide and conquer algorithm}

The divide and conquer algorithm is a method for calculating the Medial Axis Transform surface of a three-dimensional planar polyhedral object, described in [ReSt2000] and [SRX2001]. The method developed by Reddy and Turkiyyah [ReTu95] and extended by Renner and Stroud [ReSt96] is what can be termed a «constructive process». In contrast, the divide-and- 
conquer is a «divisive» technique, which starts with the exterior of the dual of the MAT and then subdivides it into separate nodes corresponding to MAT vertices.
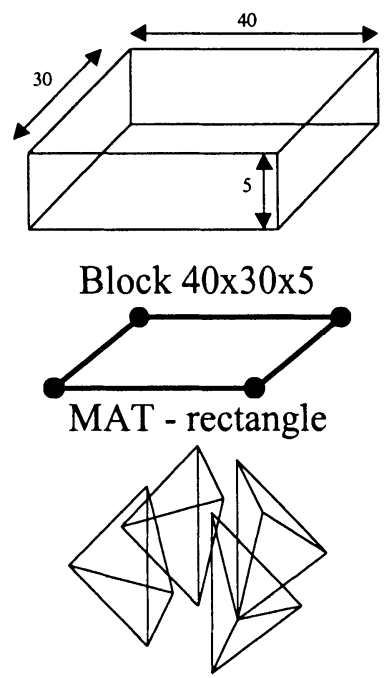

Four tetrahedral nodes

(a)

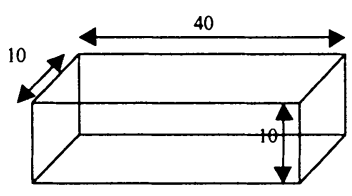

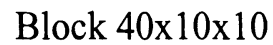
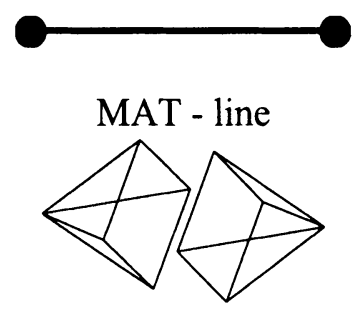

Two pyramidal nodes

(b)

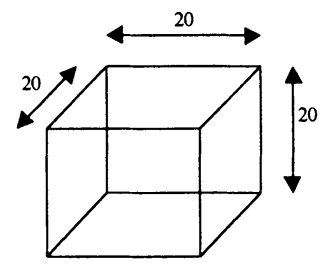

Cube 20x20x20

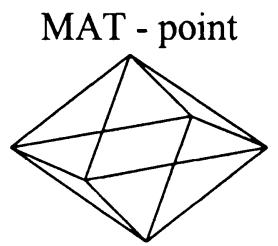

One octahedral node

(c)

Figure 1 - MAT and Delaunay nodes

This technique was termed «divide-amd-conquer» because, as the nodes are extracted, the dual structure falls into sub-pieces which are easier to subdivide, in turn, hence the MAT calculation process becomes easier, in theory. Another difference between the divide-and-conquer algorithm and the constructive processes is that the nodes, termed here «Delaunay nodes» determined in the constructive algorithm are assumed to be tetrahedra. Although the tetrahedral node is common it is only the minimum structure. Where there are degenerate parts of the object the node topology can be more complicated. To illustrate this, consider the case of a simple block in Figure 1.

The global structure, that is, topology of the union of the nodes is the same in each case, an octahedron. The different geometries of the three blocks causes the different subdivisions. In Figure la the octahedron is broken into four tetrahedra, and the MAT is a rectangle, each corner point corresponds to one of the tetrahedral nodes. In Figure $1 \mathrm{~b}$ the square cross section of the block means that there are two square pyramidal nodes and the MAT is a line. In Figure $1 \mathrm{c}$ the square block means that there is only one single node, an octahedral node, and the MAT is a single point. 
The starting point for the divide-and-conquer algorithm is the global structure. This is related to the topological dual of the object, as described in [ReSt2000] and [SRX2001]. For the cube, above, the global structure is exactly the topological dual but the topological dual has to be modified slightly to produce the global structure when there are concave edges and concave vertices in the object. The relation to the dual is because the vertices in the dual represent point touches by maximally inscribed spheres on the face, edge, or vertex. Because of this relationship it is easy to reconstruct the topology of the object, to reconstruct the offset topology of the object or to subdivide the object, as described in section 4 .

\begin{tabular}{|l|l|}
\hline Original object & Modified dual for negative object \\
\hline Delaunay node structure pour & Delaunay node structure pour \\
\hline negative MAT
\end{tabular}

Figure 2 - MAT and node structures

Figure 2 shows the steps in the creation of the MATs of positive and negative objects with the divide-and-conquer algorithm. For simplicity this is shown with a $2 \mathrm{D}$ object, the principle is the same for $3 \mathrm{D}$ objects. The first 
step is to create the dual of the object. This is then modified by adding nodes for the concave edges and vertices.

In $3 \mathrm{D}$ this is done by splitting the dual edges corresponding to concave edges of the original object. Concave vertex nodes are added by subdividing the corresponding faces in the dual, but the way in which this is omitted. The modification process is described in more detail in Stroud et al. [SRX2001]. The modified dual is the union of the Delaunay nodes corresponding to MAT vertices.

The calculation of the MAT is done by subdividing the modified dual into nodes, which depends on the nature of the geometry of the original object. The subdivided node structure is the dual of the MAT, each node corresponds to a MAT vertex. Each double edge (or double face in 3D), where the modified dual was subdivided corresponds to an edge of the MAT. The external edges (faces in 3D) of the modified dual correspond to the "wing-edges", where the radii of the maximally inscribed spheres within the object diminish to zero. Briefly the steps of the calculation process are the following:

1. Select any of the faces of the modified dual. The face represents a combination of elements (faces, concave edges and concave edges) forming one limit of a maximally inscribed sphere.

2. Find one or more extra elements, which, together with those determined in step 1, bound the maximally inscribed sphere.

3. Using the vertices corresponding to these extra elements, create and extract the topology of the Delaunay node corresponding to the maximally inscribed sphere.

4. Repeat from step 1 until every group of faces is a Delaunay node.

The calculation process is too complicated to describe in more detail here, the interested reader should refer to [ReSt2000] or [SRX2001] for details. This paper concerns the way in which the MAT information can be used for applications.

\section{The negative MAT}

Calculating the negative MAT is similar to the calculation of the positive MAT. This is done by negating the object, in effect creating an objectshaped void in a universe of material. The initial step of creating the global structure is done as before, although the actual structure is different, as illustrated in Figure 3. In the global structure for the positive object only the faces are represented as nodes whereas in the global structure for the 
negative object all the edges and the vertices are represented as nodes, since they are concave edges and concave vertices, respectively, in the negative object.

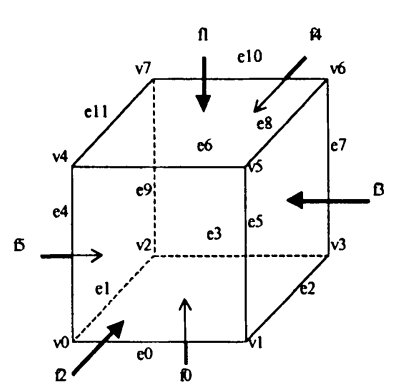

Original object

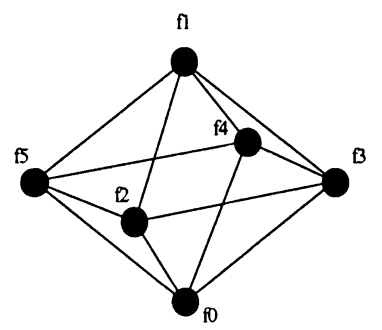

Positive global structure

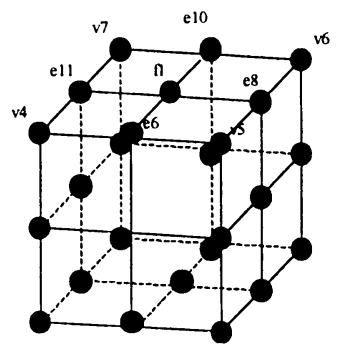

Negative structure

Figure 3 - Global structures for positive and negative MATs

There is another difference, though, in the Delaunay nodes for the negative MAT. In the positive MAT there is always an element, which acts as a delimiter for the Delaunay node, hence there is a closed topological structure for the node. In the Delaunay nodes for the negative MAT, however, there are infinite nodes, where the 'inscribed' sphere has infinite radius. With the MAT of a positive object there is always at least one extra element, but this is not necessarily true for the negative MAT. On the right hand side of Figure 2 shows the steps in the creation of the negative mat. The first step is the same as for the positive object, that is the calculation of the topological dual. The second step, the calculation of the modified dual produces a much more complicated structure than for the positive object because, in general, there are more elements for the calculation. The subdivision process creates a set of finite Delaunay nodes and a set of infinite Delaunay nodes, where no extra limiting element is found. An unproved conjecture is that the union of the bases of the infinite nodes is the convex hull of the object. This can be seen by inspection, since if the face is not on the convex hull then a sphere of finite radius will touch the base and also at least one other element, thus creating a finite Delaunay node. The vertices of the MAT of the negative object correspond to the finite Delaunay nodes, the MAT edges join adjacent nodes.

\section{Recombination methods}

One reason for studying the MAT is to be able to develop improved algorithms for applications. If the algorithms do not improve currently available software then the computationally expensive process of 
calculating the MAT is difficult to justify. On the other hand, if there are good reasons for calculating the MAT then it is likely that there will be improvements in the calculation methods which will make the MAT more attractive. The purpose of this paper is, therefore, to present MAT-based methods as a comparison for the traditional methods. The techniques described here are not part of commercial software, in fact the only methods for MAT calculation of 3D objects known to the authors are academic methods. The MAT calculation description, above, describes how the Delaunay nodes are created by subdivision. This section describes methods for recombining the Delaunay nodes and how this can be used for applications. It is known that 2D Voronoi methods can be used for the calculation of toolpaths for pocket machining. The methods described here can be used for offsetting and analysis in 3D.

\subsection{Offsetting}

Object offsetting can be performed relatively easily and quickly using the Delaunay nodes. The Delaunay node subdivision provides information about the interactions between boundary elements. If the object is offset afresh for a set of values then, for each offset value, it is necessary to perform global checks, a sort of Boolean operation, for each value to find the final structure. There is, therefore, a trade-off between the cost of the initial calculation of the Delaunay nodes and the cost of separate offsetting. The MAT-based offsetting method, described here, allows the user to experiment with different values to determine the cutter centre surfaces. In addition to the offsetting process, the radii of the maximally inscribed spheres provide information about the critical offsets, where the topology of the offset object changes. This information indicates the range of tool sizes that can be used for machining different parts of the object.

Figure 4 shows recombination of the Delaunay nodes for offsetting the positive object. The set of the nodes together with the radii of the maximally inscribed sphere is show in the first figure. Column (a) shows the case where the offset is less than four. Column (b) shows the case where the offset is four or more, but less than six. Column (c) shows the case where the offset is six or more, but less than ten. The first line of the figure shows the nodes available for recombination, that is, nodes with associated radius greater than the offset value. The second line of the figure shows the recombined nodes and the last line the offset geometry. In the first column, with the lowest range of offsets all the Delaunay nodes are available for recombination, creating a figure with almost the same topology as the original figure, the only difference being that two circular arcs are added corresponding to the concave figures. The geometry is found by offsetting 
the geometry of the original object. Edge curves are offset directly, the offset of a vertex is a circular arc. If the offset is zero, though, then the corresponding arc has zero length and is replaced by a vertex. For the second column, with $4 \leq$ offset $<6$ the two small triangular nodes (nodes $\mathrm{A}$ and $\mathrm{D}$ ) are ignored and the topology of the resulting object is the dual of the union of nodes $\mathrm{B}, \mathrm{C}$ and $\mathrm{E}$. In effect this means that the circular arcs corresponding to the concave vertices cancel out the two short side edges. In the final figure only node $\mathrm{E}$ remains and the topology of the final offset figure is a pentagon.

The offsetting process for the negative MAT is the same, involving a size filter of the Delaunay nodes, node joining and geometric reconstruction, as shown in Figure 5. In effect the external offset is the internal offset of the object shaped void in the infinite object. The infinite Delaunay nodes, represented by lines in the figure have a notional common element at infinity and so are infinite pyramids.
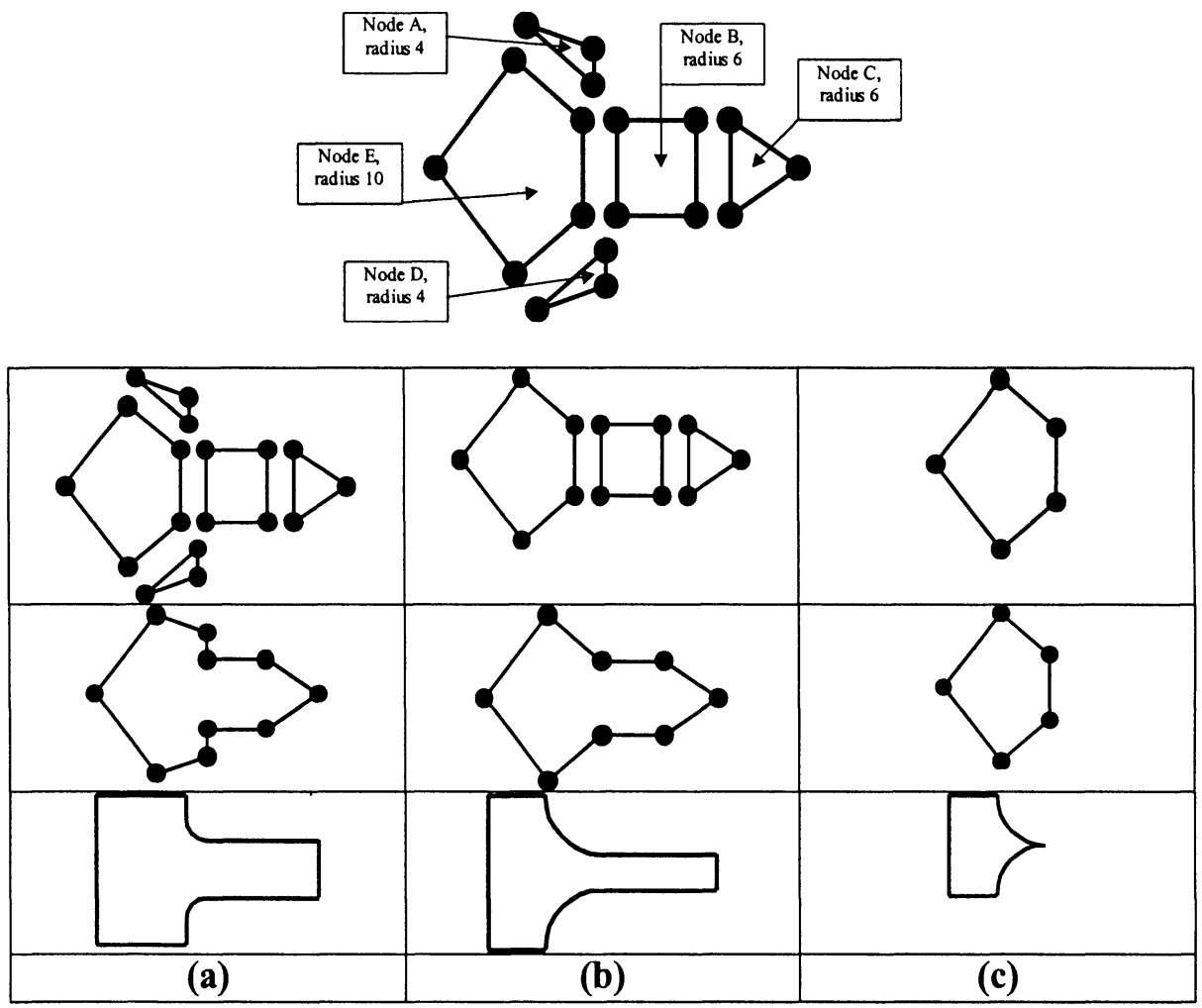

Figure 4 - Delaunay node recombination for offsetting a positive object 
The first column shows the case for offsets less than four. In the node recombination step the double edges essentially cancel each other out. The figure produced is similar to the original shape, but circular arcs are introduced for each vertex except the two originally concave vertices. The second column shows the case for $4 \leq$ offset $<62.5$. As before, the short side edges disappear because the circular arcs corresponding to one of the end vertices cancel out the edge. In the final column, for an offset $\geq 62.5$ the top and bottom edges of the side protrusion disappear, leaving the final offset figure.
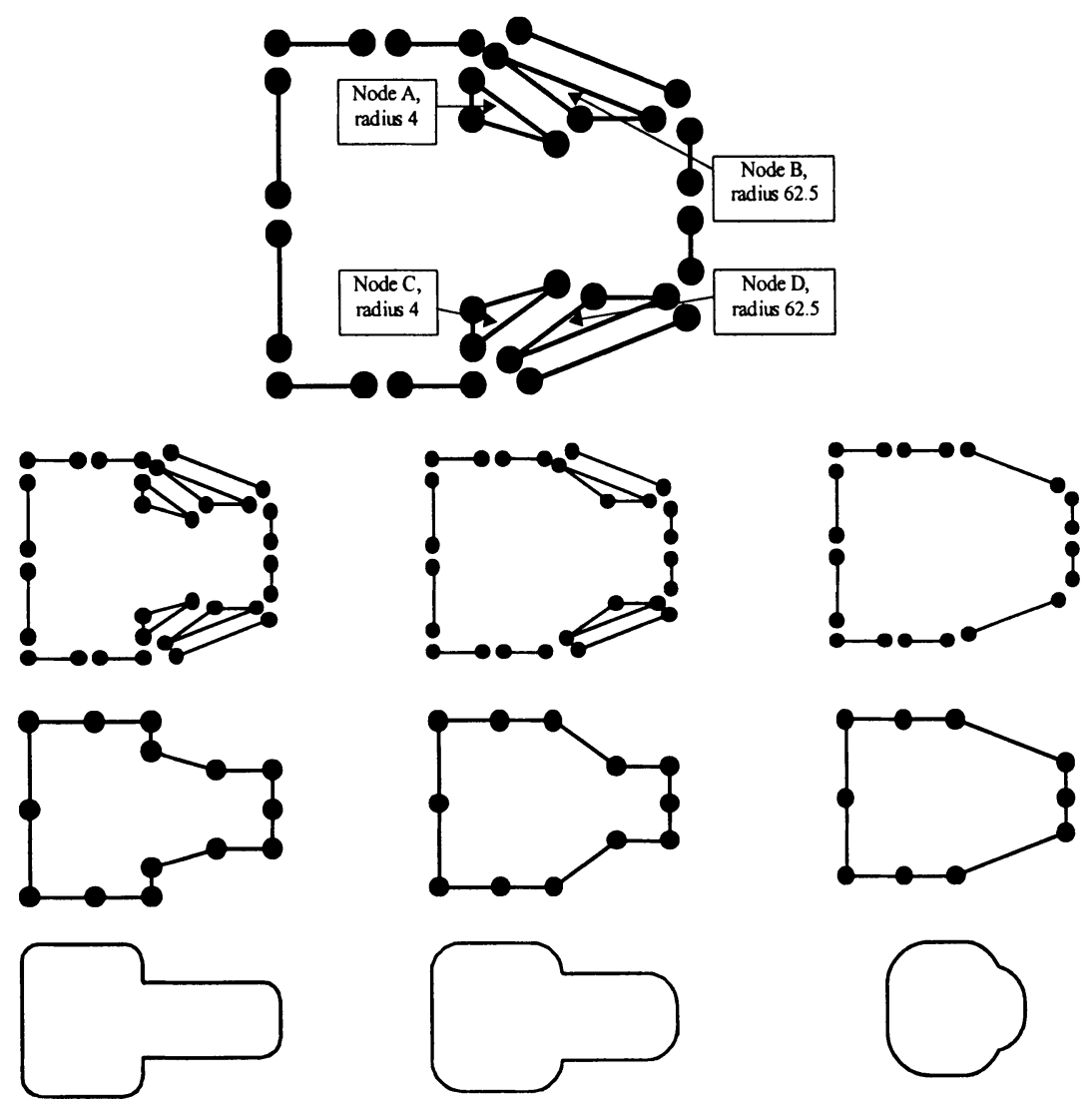

Figure 5 - Recombination for offsetting of a negative object

The above examples are simple 2D examples to show the working of the Delaunay node creation and recombination processes. Figure 6 shows a 3D example created in the same way. In addition the node size information can be used to create offsets where nodes disappear, that is, where the topology of the offset structure changes. The image on the right is the view from 
above to show these changes. The critical offset values are, for this object: $3.375,6.75,10.0,15.0$ and 15.358984 .

\subsection{Volumetric decomposition}

The offsetting procedure uses one set of rules for recombination. Another set of rules gives a volumetric decomposition method, as illustrated in Figure 7.

The Delaunay nodes are joined without regard for their size, but are not joined if one of the vertices of the face corresponds to a concave edge limiting a face of the node. Such a set of simple rules was developed for the object above. More complex rules for general cases are still to be developed.This technique can be used for decomposition of delta volumes, say, to determine volumetric regions to be removed in the machining process.
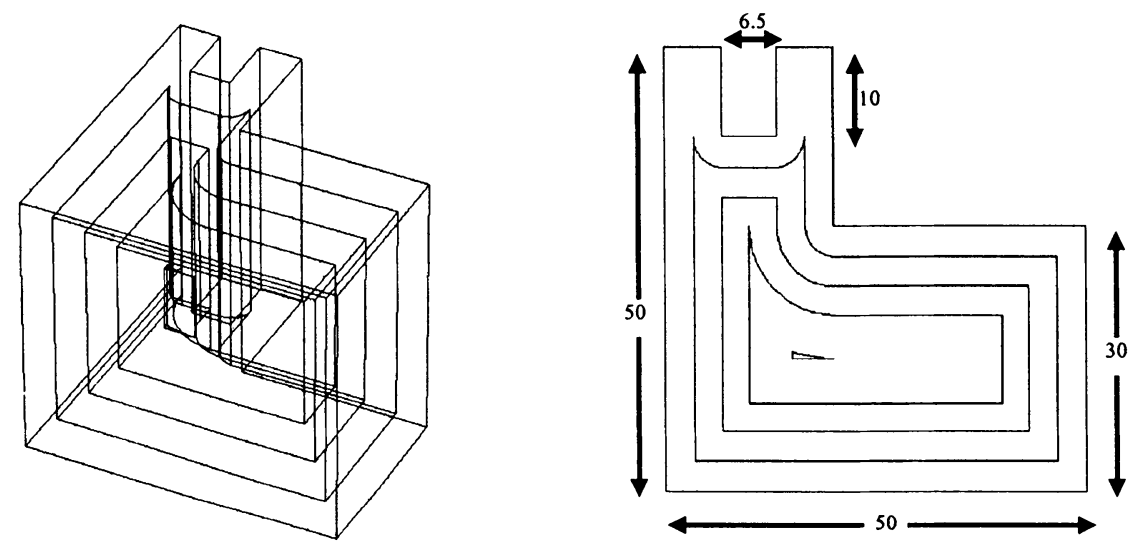

Figure 6 - Topological change offsets in 3D
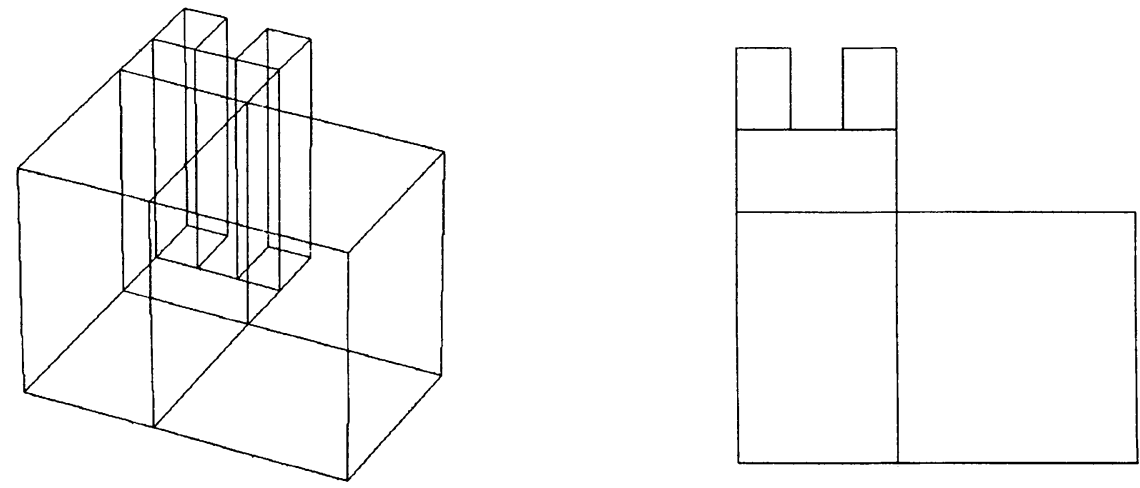


\section{Conclusions}

This paper describes MAT-based techniques for offsetting and decomposing three dimensional objects. The basis for the method is the divide-andconquer MAT algorithm for calculating general topology Delaunay nodes which can then be recombined to produce the offset or decomposition. MAT-based applications are still in their infancy and need to be developed further in order to see how the MAT can be used. The offsetting technique presented here is useful, for example, if multiple offsets are to be created. The proximity calculations are calculated once, in the form of a MAT structure, and are then reused.

\section{References}

[ReTu95] Reddy, J., Turkiyyah, G. (1995), "Computation of 3d skeletons by a generalised Delaunay triangulation technique", $\mathrm{CAD}, \mathrm{pp}$. 677-694.

[ReSt96] Renner, G., Stroud, I. (1996) "Medial surface generation and refinement", in "Product Modelling for Computer Integrated Design and Manufacture", ed. Pratt, Sriram, Wozny, pub. Chapman \& Hall 1997, ISBN/ISSN: 0-412-80980-

[ReSt2000] Renner, G., Stroud, I. (2000) "Techniques for the calculation of medial surfaces of solids", submitted to CAD Journal

[SAR95] Sheehy, D. J., Armstrong, C. G., Robinson, D. J. (1995), Computing the Medial Surface of a Solid from a Domain Delaunay Triangulation, in. Proc. Third Symp. on Solid Modeling and Appl., ed. C.M.Hoffmann and J.R.Rossignac, ACM, pp 201-212.

[SPB95] Sherbrooke, E. C., Patrikalakis, N. M., Brisson, E. (1995), "Computation of the Medial Axis Transform of 3-D Polyhedra", in Proc.Third Symposium on Solid Modeling and Applications, ed. C.M.Hoffmann and J.R.Rossignac, pub. ACM, pp. 187-199.

[SRX2001] Stroud, I.A., Renner, G., Xirouchakis, P. "A divide and conquer algorithm for medial surface calculation", in preparation. 